\title{
Fock-Sobolev 空间上有界、紧与 $S_{p^{-}}$类算子
}

\author{
王晓峰，夏锦*，曹广福 \\ 广州大学数学与信息科学学院, 广州 510006 \\ E-mail: wangxiaofeng514@hotmail.com,xiaj@cdut.edu.cn, guangfucao@163.com \\ 收稿日期: 2012-12-31；接受日期: 2013-08-14; * 通信作者 \\ 国家自然科学基金 (批准号: 11271092 和 11301101) 和广州市教育局高校科技计划 (批准号: 2012A018) 资助项目
}

摘要 本文研究 Fock-Sobolev 空间上稠密定义算子, 将这些算子统一表示成积分算子, 利用积分算子 的方法得到了它们的一个充分条件, 并构造反例说此充分条件是非必要的, 还得到这些算子为紧算子 的两个充分条件.最后构造符号函数在复平面上每一点处本性无界的紧和 $S_{p^{-}}$类 $(0<p<\infty)$ Toeplitz 算子.

关键词 Fock-Sobolev 空间 Toeplitz 算子 有界性 紧性 Schatten $p$ - 类

MSC (2010) 主题分类 $30 \mathrm{H} 20,47 \mathrm{~B} 07,47 \mathrm{~B} 35,47 \mathrm{~B} 38$

\section{1 引言}

设 $\mathbb{C}$ 表示复平面, $d A$ 表示 $\mathbb{C}$ 上的 Lebesgue 面积测度. $F^{2}$ 是由 $L^{2}\left(\mathbb{C}, \mathrm{e}^{-|z|^{2}} d A(z)\right)$ 中整函数所构 成的空间, 于是,

$$
\|f\|_{2}^{2}=\left(\frac{1}{\pi} \int_{\mathbb{C}}|f(z)|^{2} \mathrm{e}^{-|z|^{2}} d A(z)\right), \quad \forall f \in F^{2} .
$$

对任意给定的正整数 $m$, Fock-Sobolev 空间 $F^{2, m}$ 由 $m$ 阶导函数属于 $F^{2}$ 的整函数构成, 故 $F^{2, m}$ 的 范数为

$$
|f(0)|+\cdots+\left|f^{(m-1)}(0)\right|+\left\|f^{(m)}\right\|_{2}, \quad \forall f \in F^{2, m} .
$$

$\mathrm{Cho}$ 和 $\mathrm{Zhu}{ }^{[1]}$ 证明了 $f \in F^{2, m}$ 当且仅当 $z^{m} f(z) \in F^{2}$. 因此, 在 $F^{2, m}$ 中也可以用下述范数:

$$
\|f\|_{2, m}^{2}=c(2, m) \int_{\mathbb{C}}\left|z^{m} f(z) \mathrm{e}^{-\frac{|z|^{2}}{2}}\right|^{2} d A(z), \quad \forall f \in F^{2, m},
$$

其中 $c(2, m)=\frac{1}{\pi m !}$ 是一个常数, 它使得常值函数 1 在 $F^{2, m}$ 中范数刚好为 1 . 由开映射定理有

$$
\|f\|_{2, m} \sim|f(0)|+\cdots+\left|f^{(m-1)}(0)\right|+\left\|f^{(m)}\right\|_{2} .
$$

为使用方便, 本文将使用 $\|\cdot\|_{2, m}$ 为 $F^{2, m}$ 的范数. 在本文中使用记号 $\|\cdot\|_{p, m}$ 表示如下范数:

$$
\|f\|_{p, m}^{p}=\frac{1}{\pi m !} \int_{\mathbb{C}}|f(z)|^{p} \mathrm{e}^{-|z|^{2}}|z|^{2 m} d A(z) .
$$


由定义知, Fock-Sobolev 空间 $F^{2, m}$ 是 Hilbert 空间, 其上内积为

$$
\langle f, g\rangle_{m}=\int_{\mathbb{C}} f(z) \overline{g(z)} \mathrm{e}^{-|z|^{2}}|z|^{2 m} d A(z) .
$$

直接计算知, $F^{2, m}$ 的正规正交基为 (参见文献 [1])

$$
e_{n}(z)=\sqrt{\frac{\Gamma(m+1)}{\Gamma(n+m+1)}} z^{n}=\sqrt{\frac{m !}{(n+m) !}} z^{n}, \quad n=0,1,2, \ldots
$$

故 $F^{2, m}$ 的再生核为

$$
K_{m}(z, w)=\sum_{n=0}^{\infty} \frac{m !}{(n+m) !}(z \bar{w})^{n}=\frac{m !\left(\mathrm{e}^{z \bar{w}}-p_{m}(z \bar{w})\right)}{(z \bar{w})^{m}},
$$

其中 $p_{m}(z \bar{w})=\sum_{n=0}^{m-1} \frac{(z \bar{w})^{n}}{n !}$ 是 $\mathrm{e}^{z \bar{w}}$ 的 Taylor 展开式的前 $m-1$ 项.

下文用 $L_{m}^{2}$ 表示可测函数空间 $L^{2}\left(\mathbb{C},|z|^{2 m} \mathrm{e}^{-|z|^{2}} d A(z)\right) . L_{m}^{2}$ 的范数为

$$
\|f\|_{2, m}^{2}=c(2, m) \int_{\mathbb{C}}\left|z^{m} f(z) \mathrm{e}^{-\frac{|z|^{2}}{2}}\right|^{2} d A(z) .
$$

易知正交投影 $P_{m}: L_{m}^{2} \rightarrow F^{2, m}$ 是积分算子

$$
P_{m} f(w)=\frac{1}{m ! \pi} \int_{\mathbb{C}} f(z) K_{m}(w, z) \mathrm{e}^{-|w|^{2}}|z|^{2 m} d A(z),
$$

其中 $K_{m}(z, w)=K_{m, w}(z)$ 是 $F^{2, m}$ 的再生核. 由文献 [1] 知, $P_{m}$ 是从 $L_{m}^{2}$ 到 $F^{2, m}$ 上的有界线性算子.

若 $\mu$ 是 $\mathbb{C}$ 上复 Borel 测度, 定义 Toeplitz 算子 $T_{\mu}$ 为

$$
T_{\mu}(f)(w)=\int_{\mathbb{C}} f(z) K_{m}(w, z) \mathrm{e}^{-|z|^{2}}|z|^{2 m} d \mu(z), \quad z \in \mathbb{C}, \quad f \in F^{2, m} .
$$

为了使 $(1.1)$ 中积分收玫, 要求 $\mu$ 满足

$$
\int_{\mathbb{C}}\left|K_{m}(w, z)\right|^{2} \mathrm{e}^{-|z|^{2}}|z|^{2 m} d|\mu|(z)<\infty, \quad \forall w \in \mathbb{C} .
$$

若 $\mu$ 满足 (1.2) 的条件, 因 $F^{2, m}$ 的再生核函数的有限线性组合在 $F^{2, m}$ 中稠密, Toeplitz 算子 $T_{\mu}$ 在 $F^{2, m}$ 上稠定义.

人们研究 Fock 空间上算子已经有很长一段时间了. 许多的数学家和物理学家都很关注 Fock 空 间上算子. Berger 和 Coburn ${ }^{[2-4]}$ 讨论了 Fock 空间上 Toeplitz 算子. Coburn 等人 ${ }^{[5]}$ 研究了 Fock 空 间上以 BMO (有界均值震荡) 函数为符号的 Toeplitz 算子. Isralowitz 和 Zhu ${ }^{[6]}$ 给出了 Fock 空间上 以正函数为符号 Toeplitz 算子是有界、紧和 $S_{p^{-}}$类算子的充要条件. Zhu ${ }^{[7]}$ 系统地介绍了 Fock 空间 上 Toeplitz 算子、小 Hankel 算子和 Hankel 算子的性质. Fock-Sobolev 空间上算子的性质是一个新的 课题, 它最先由 Cho 和 Zhu ${ }^{[1]}$ 引入和研究. 后来, Schuster 和 Varolin ${ }^{[8]}$ 刻画了广义 Bargmann-Fock 空间上有界和紧 Toeplitz 算子. Cho, Isralowitz 和 Joo 等算子专家现在也很关注并且正在研究广义加 权 Fock 空间上 Toeplitz 算子.

本文将使用条件 $\left\|U_{z} S k_{m, z}\right\|_{p}<C$ 去刻画 Fock-Sobolev 空间上算子 $S$ 的有界性和紧性, 其中

$$
U_{a} f(z)=f(a-z) \mathrm{e}^{-\frac{|a|^{2}}{2}+z \bar{a}} \frac{(a-z)^{m}}{z^{m}}
$$


是 $F^{2, m}$ 上的西算子, $k_{m, z}$ 是 $F^{2, m}$ 的正规化再生核. 事实上, 条件 $\left\|U_{z} S k_{m, z}\right\|_{p}<C$ 是条件 $\left\|S_{z} 1\right\|_{p}<C$ 的代替品, 其中 $C$ 为常数. Axler 和 Zheng ${ }^{[9]}$ 首次使用 $\left\|S_{z} 1\right\|_{p}<C$ 来刻画 Bergman 空间上算子 $S$ 有 界性和紧性. 在文献 [5,10-13] 中, 此条件得到了更进一步的讨论. 因 Fock-Sobolov 空间比 Fock 空间 更复杂, 条件 $\left\|S_{z} 1\right\|_{p}<C$ 不再适用, 故本文引入 $\left\|U_{z}^{m} S k_{m, z}\right\|_{p}<C$ 去研究 Fock-Sobolov 空间上算子 的有界性和紧性.

第 2 和 3 节的证明中应用了文献 $[9,10,12]$ 的思想,得到了算子是有界和紧算子的充分条件,但没 有使用 Schur 检验. 本文对 $p>2$ 的情形得到了 Fock-Sobolov 空间上算子的有界性和紧性, 此结果与 Bergman 空间情形有较大不同. 本文的证明比文献 [10] 中的证明更难更复杂.

若 $T_{\psi}$ 是 Hardy 空间上 Toeplitz 算子, 则 $T_{\psi}$ 有界当且仅当 $\psi$ 是本性有界, $T_{\psi}$ 紧当且仅当 $\psi=0$. 但在 Bergman 空间和 Dirichlet 空间上却存在许多符号本性无界的有界, 甚至紧 Toeplitz 算子. Cima 和 Cuckovic ${ }^{[14]}$ 利用 Cantor 集构造了一类函数使得以这些函数为符号的 Toeplitz 算子为紧算子. Cao ${ }^{[15]}$ 构造了 Bergman 空间上以复单位球面上本性无界函数为符号的迹类 Toeplitz 算子. Xia 等人 ${ }^{[16]}$ 甚 至构造出了 Dirichlet 空间上以复单位球面本性无界函数为符号的 Schatten $p$ - 类 Toeplitz 算子, 其中 $0<p<\infty$. 第 4 节构造 Fock-Sobolev 空间 $F^{2, m}$ 上符号函数在复平面上每点处本性无界的 Schatten $p$ 类 Toeplitz 算子, 其中 $0<p<\infty$.

\section{2 有界性}

本节将刻画 Fock-Sobolev 空间上算子的有界性. 下面的引理给出一个在后文中多次使用的重要 的逐点估计.

引理 1 设 $f$ 是 $L^{p}\left(\mathbb{C}, \frac{1}{2 \pi m !} \mathrm{e}^{-|z|^{2}}|z|^{2 m} d A(z)\right)$ 中的整函数, 则对任意 $p>0, z \in \mathbb{C}$ 有

$$
|f(z)| \leqslant C\|f\|_{p, m} \mathrm{e}^{\frac{|z|^{2}}{p}}|z|^{-\frac{2 m}{p}},
$$

其中 $C$ 为常数.

证明 由定义得

$$
\|f\|_{p, m}^{p}=\frac{1}{\pi m !} \int_{\mathbb{C}}|f(z)|^{p} \mathrm{e}^{-|z|^{2}}|z|^{2 m} d A(z)=\frac{1}{\pi m !} \int_{\mathbb{C}}\left|f(z) \mathrm{e}^{-\frac{|z|^{2}}{p}} z^{\frac{2 m}{p}}\right|^{p} d A(z) \geqslant C|f(z)|^{p}|z|^{2 m} \mathrm{e}^{-|z|^{2}},
$$

其中 $C>0$ 为常数, 上面最后一个不等式参见文献 [7, 推论 2.8]. 于是, $|f(z)| \leqslant C\|f\|_{p, m} \mathrm{e}^{\frac{|z|^{2}}{p}}|z|^{-\frac{2 m}{p}}$. 引理证毕.

下面对任意 $a \in \mathbb{C}$ 引入 $F^{2, m}$ 上一个酉算子 $U_{a}$, 其定义如下:

$$
U_{a} f(z)=f(a-z) \mathrm{e}^{-\frac{|a|^{2}}{2}+z \bar{a}} \frac{(a-z)^{m}}{z^{m}} .
$$

算子 $U_{a}$ 是 Fock 空间上西算子 $U_{a} f(z)=f(a-z) \mathrm{e}^{-\frac{|a|^{2}}{2}+z \bar{a}}$ 在 $F^{2, m}$ 上的修订版本.

下面的结论在本节主要结论的证明中有重要作用.

引理 2 对任意 $p>2$ 和 $F^{2, m}$ 上线性算子 $S$ 有

$$
\begin{aligned}
\left|\left\langle S K_{m, w}, K_{m, z}\right\rangle_{m}\right| \leqslant & C_{1}\left\|U_{w} S k_{m, w}(z)\right\|_{p, m}|z-w|^{2 \sigma}|z|^{-m}|w|^{-m} \\
& \times\left(\mathrm{e}^{\frac{1}{2}\left(|z|^{2}+|w|^{2}\right)-\sigma|z-w|^{2}}+\mathrm{e}^{\frac{|z|^{2}}{2}-\sigma|z-w|^{2}} \sqrt{p_{m}\left(|w|^{2}\right)}\right),
\end{aligned}
$$


其中 $w, z \in \mathbb{C}, \sigma=\frac{1}{2}-\frac{1}{p}, C_{1}$ 为常数. 进而, 若 $\left\|U_{w} S k_{m, w}\right\|_{p} \leqslant C_{2}$, 其中常数 $C_{2}>0, w \in \mathbb{C}$, 则存在常 数 $C$ 使得对任意 $z, w \in \mathbb{C}$ 有

$$
\left|\left\langle S K_{m, w}, K_{m, z}\right\rangle_{m}\right| \leqslant C|z-w|^{2 \sigma}|z|^{-m}|w|^{-m}\left(\mathrm{e}^{\frac{1}{2}\left(|z|^{2}+|w|^{2}\right)-\sigma|z-w|^{2}}+\mathrm{e}^{\frac{|z|^{2}}{2}-\sigma|z-w|^{2}} \sqrt{p_{m}\left(|w|^{2}\right)}\right) .
$$

证明 由 $U_{w}$ 的定义知,

$$
\left(U_{w} S k_{m, w}\right)(z)=\mathrm{e}^{-\frac{1}{2}\left(|w-z|^{2}-|z|^{2}\right)} \frac{|w-z|^{m}}{|z|^{m}}\left(S k_{m, w}\right)(w-z) .
$$

于是, 由引理 1 得

$$
\left|\left(S k_{m, w}\right)(w-z)\right| \leqslant C_{3}\left\|U_{w} S k_{m, w}\right\|_{p, m} \mathrm{e}^{\frac{1}{2}|w-z|^{2}-\sigma|z|^{2}} \frac{|z|^{2 m \sigma}}{|w-z|^{m}}, \quad \forall z, w \in \mathbb{C},
$$

其中 $\sigma=\frac{1}{2}-\frac{1}{p}$. 在上式中用 $w-z$ 代替 $z$ 得

$$
\left|\left(S k_{m, w}\right)(z)\right| \leqslant C_{3}\left\|U_{w} S k_{m, w}\right\|_{p, m} \mathrm{e}^{\frac{1}{2}|z|^{2}-\sigma|w-z|^{2}} \frac{|w-z|^{2 m \sigma}}{|z|^{m}} .
$$

在前式中将 $\left(S k_{m, w}\right)(z)$ 用下式替换:

$$
S k_{m, w}(z)=\frac{|w|^{m}}{\sqrt{\mathrm{e}^{|w|^{2}}-p_{m}\left(|w|^{2}\right)}} S K_{m, w}(z)=\frac{|w|^{m}}{\sqrt{\mathrm{e}^{|w|^{2}}-p_{m}\left(|w|^{2}\right)}}\left\langle S K_{m, w}, K_{m, z}\right\rangle_{m},
$$

并化简得

$$
\begin{aligned}
\left|\left\langle S K_{m, w}, K_{m, z}\right\rangle_{m}\right| \leqslant & C_{3}\left\|U_{w} S k_{m, w}\right\|_{p, m}|z-w|^{2 \sigma}|z|^{-m}|w|^{-m}\left(\mathrm{e}^{\frac{1}{2}\left(|z|^{2}+|w|^{2}\right)-\sigma|z-w|^{2}}\right. \\
& \left.+\mathrm{e}^{\frac{|z|^{2}}{2}-\sigma|z-w|^{2}} \sqrt{p_{m}\left(|w|^{2}\right)}\right), \quad \forall z, w \in \mathbb{C} .
\end{aligned}
$$

引理证毕.

在下面的引理中, 将使用积分算子的形式表示 $F^{2, m}$ 上所有算子. 利用此表示可以得到 $F^{2, m}$ 上算 子的有界性.

引理 3 对 $F^{2, m}$ 上任意线性算子 $S$, 若 $T$ 是如下定义的 $L^{2}\left(\mathbb{C}, d \lambda_{m}\right)$ 上的积分算子

$$
T f(z)=\int_{\mathbb{C}} f(w)\left\langle S K_{m, w}, K_{m, z}\right\rangle_{m} d \lambda_{m}(w),
$$

其中 $d \lambda_{m}=\frac{1}{\pi m !} \mathrm{e}^{-|z|^{2}}|z|^{2 m} d A(z)$, 则 $S$ 在 $F^{2, m}$ 上有界当且仅当 $T$ 在 $L^{2}\left(\mathbb{C}, d \lambda_{m}\right)$ 上有界. 实际上, 当 $S$ 或 $T$ 有界时, $S$ 是 $T$ 在 $F^{2, m}$ 上的限制.

证明 易知对任意 $z \in \mathbb{C}$, 函数

$$
w \mapsto\left\langle K_{m, z}, S K_{m, w}\right\rangle_{m}=\left\langle S^{*} K_{m, z}, K_{m, w}\right\rangle_{m}=\left(S^{*} K_{m, z}\right)(w)
$$

为整函数且属于 $F^{2, m}$, 故对任意 $f \in L^{2}\left(\mathbb{C}, d \lambda_{m}\right) \ominus F^{2, m}$ 有 $T f=0$.

设 $S$ 为 $F^{2, m}$ 上有界算子且 $f=K_{m, a}$ 为点 $a \in \mathbb{C}$ 处的再生核函数, 那么, 由 $K_{m, a}$ 的再生性质得

$$
\begin{aligned}
T f(z) & =\frac{\int_{\mathbb{C}} K_{m}(w, a)\left\langle S K_{m, w}, K_{m, z}\right\rangle_{m} d \lambda_{m}(w)}{} \\
& =\int_{\mathbb{C}}\left\langle S^{*} K_{m, z}, K_{m, w}\right\rangle_{m} K_{m}(a, w) d \lambda_{m}(w)
\end{aligned}
$$




$$
\begin{aligned}
& =\overline{\left\langle S^{*} K_{m, z}, K_{m, a}\right\rangle_{m}}=\left\langle S K_{m, a}, K_{m, z}\right\rangle_{m} \\
& =S K_{m, a}(z)=S f(z) .
\end{aligned}
$$

这表明在 $\mathcal{F}$ 上 $T f=S f$ 且对全体 $f \in \mathcal{F}$ 有 $\|T f\|_{2, m} \leqslant\|S\|\|f\|_{2, m}$, 其中 $\mathcal{F}$ 是 $F^{2, m}$ 中全体再生核的 有限线性组合所成集合, $\mathcal{F}$ 中函数可表示为

$$
f(z)=\sum_{k=1}^{N} c_{k} K_{m, w_{k}}(z), \quad \forall f \in \mathcal{F} .
$$

众所周知, $\mathcal{F}$ 是 $F^{2, m}$ 的稠密子集 ${ }^{[7]}$. 由 $(2.2)$ 和 $\mathcal{F}$ 的定义, 很明显, $T$ 是 $L^{2}\left(\mathbb{C}, d \lambda_{m}\right)$ 上有界算子且 $S$ 为 $T$ 在 $F^{2, m}$ 上限制.

另一方面, 设 $T$ 在 $L^{2}\left(\mathbb{C}, d \lambda_{m}\right)$ 上有界且 $f \in \mathcal{F}$, 则对任意 $z \in \mathbb{C}$ 有

$$
T f(z)=\int_{\mathbb{C}} f(w) \overline{S^{*} K_{m, z}(w)} d \lambda_{m}(w)=\left\langle f, S^{*} K_{z}\right\rangle_{m}=\left\langle S f, K_{m, z}\right\rangle_{m}=S f(z) .
$$

由于 $T$ 在 $\mathcal{F}$ 上限制与 $S$ 在 $\mathcal{F}$ 上作用相同, 又因 $\mathcal{F}$ 在 $F^{2, m}$ 中稠密且 $T$ 有界, 所以, $S$ 可以延拓 为 $F^{2, m}$ 上有界算子.

接下来证明本节的主要结论.

定理 4 设 $S$ 为 $F^{2, m}$ 上线性算子, 且存在 $p>2$ 和 $C>0$ 使得对任意 $z \in \mathbb{C}$ 有 $\left\|U_{z} S k_{m, z}\right\|_{p, m}$ $\leqslant C$, 则 $S$ 在 $F^{2, m}$ 上有界, 且有 $\|S\| \leqslant\left(p C^{\prime}\right) /(p-2)$.

证明 由引理 3 知, 只需证明 $(2.1)$ 中定义的积分算子 $T$ 在 $L^{2}\left(\mathbb{C}, d \lambda_{m}\right)$ 上有界.

对引理 3 中同样常数 $C$ 与

$$
\sigma=\frac{1}{2}-\frac{1}{p}
$$

引理 2 表明, 对任意 $z \in \mathbb{C}$ 有

$$
|T f(z)| \leqslant C \int_{\mathbb{C}}|f(w)||z-w|^{2 \sigma}|z|^{-m}|w|^{-m} \mathrm{e}^{-\sigma|z-w|^{2}}\left(\mathrm{e}^{\frac{1}{2}\left(|z|^{2}+|w|^{2}\right)}+\mathrm{e}^{\frac{|z|^{2}}{2}} \sqrt{p_{m}\left(|w|^{2}\right)}\right) d \lambda_{m}(w) .
$$

令

$$
F(z)=|T f(z)| \mathrm{e}^{-\frac{1}{2}|z|^{2}}|z|^{m}
$$

由 $(2.3)$ 和 $F(z)$ 的定义知,

$$
F(z) \leqslant C_{1} \int_{\mathbb{C}}|f(w)||z-w|^{2 \sigma}|w|^{m} \mathrm{e}^{-\sigma|z-w|^{2}}\left(\mathrm{e}^{-\frac{1}{2}|w|^{2}}+\mathrm{e}^{-|w|^{2}} \sqrt{p_{m}\left(|w|^{2}\right)}\right) d A(w),
$$

其中 $C_{1}=\frac{C}{m ! \pi}$ 为常数. 故明显有

$$
\begin{aligned}
F(z)^{2} \leqslant & C_{1}^{2} \int_{\mathbb{C}}|f(w)|^{2}|w|^{2 m}\left(\mathrm{e}^{-\frac{1}{2}|w|^{2}}+\mathrm{e}^{-|w|^{2}} \sqrt{p_{m}\left(|w|^{2}\right)}\right)^{2} \\
& \times|z-w|^{2 \sigma} \mathrm{e}^{-\sigma|z-w|^{2}} d A(w) \int_{\mathbb{C}}|z-w|^{2 \sigma} \mathrm{e}^{-\sigma|z-w|^{2}} d A(w) \\
= & C_{2} \int_{\mathbb{C}}|f(w)|^{2}|w|^{2 m}\left(\mathrm{e}^{-\frac{1}{2}|w|^{2}}+\mathrm{e}^{-|w|^{2}} \sqrt{p_{m}\left(|w|^{2}\right)}\right)^{2}|z-w|^{2 \sigma} \mathrm{e}^{-\sigma|z-w|^{2}} d A(w),
\end{aligned}
$$

其中 $C_{2}=C_{1}^{2} \int_{\mathbb{C}}|z-w|^{2 \sigma} \mathrm{e}^{-\sigma|z-w|^{2}} d A(w)$. 
由 Fubini 定理和变量替换得

$$
\begin{aligned}
\int_{\mathbb{C}}\left|T f(z) \mathrm{e}^{-\frac{1}{2}|z|^{2}} z^{m}\right|^{2} d A(z) \leqslant & C_{2} \int_{\mathbb{C}}|f(w)|^{2}|w|^{2 m} \mathrm{e}^{-|w|^{2}} d A(w) \int_{\mathbb{C}}|z-w|^{2 \sigma} \mathrm{e}^{-\sigma|z-w|^{2}} d A(z) \\
& +C_{2} \int_{\mathbb{C}}|f(w)|^{2}|w|^{2 m} \mathrm{e}^{-2|w|^{2}} p_{m}\left(|w|^{2}\right) d A(w) \int_{\mathbb{C}}|z-w|^{2 \sigma} \mathrm{e}^{-\sigma|z-w|^{2}} d A(z) .
\end{aligned}
$$

因存在常数 $C_{3}$ 使得

$$
\begin{aligned}
& \int_{\mathbb{C}}|f(w)|^{2}|w|^{2 m} \mathrm{e}^{-2|w|^{2}} p_{m}\left(|w|^{2}\right) d A(w) \leqslant C_{3} \int_{\mathbb{C}}|f(w)|^{2}|w|^{2 m} \mathrm{e}^{-|w|^{2}} d A(w), \\
& \int_{\mathbb{C}}|z-w|^{2 \sigma} \mathrm{e}^{-\sigma|z-w|^{2}} d A(z)<C_{4} \frac{p}{p-2},
\end{aligned}
$$

于是有

$$
\int_{\mathbb{C}}\left|T f(z) \mathrm{e}^{-\frac{1}{2}|z|^{2}} z^{m}\right|^{2} d A(z) \leqslant C_{5} \frac{p}{p-2} \int_{\mathbb{C}}|f(w)|^{2}|w|^{2 m} \mathrm{e}^{-|w|^{2}} d A(w),
$$

其中 $C_{5}$ 为常数. 这说明 $T$ 在 $L^{2}\left(\mathbb{C}, d \lambda_{m}\right)$ 上有界且

$$
\|T\| \leqslant C_{5} \frac{p}{p-2} .
$$

将 $T$ 限制到 $F^{2, m}$ 则得到 $S$ 的有界性.

一个自然的问题是 $F^{2, m}$ 上算子有界的充分必要条件是什么? $\left\|U_{z} S k_{m, z}\right\|_{2}<C$ 是比较合适的条 件. 但不幸的是, 现在没有任何办法证明 $\left\|U_{z} S k_{m, z}\right\|_{2}<C$ 为 $S$ 有界的充分条件. 下面的例子将说明 $\left\|U_{z} S k_{m, z}\right\|_{p}<C$ 离必要条件差别很远. 这个例子与 Cao 等人 ${ }^{[10]}$ 的思想很接近.

若 $p>2$, 因为 $1>\left(\frac{p}{4}-1\right)$, 故可以找到正数 $r$ 使得

$$
1>r>\left(\frac{p}{4}-1\right) .
$$

对前面给定的 $r$, 设 $S$ 为 $F^{2, m}$ 上线性算子, 其定义为 $S(f)(w)=f(-r w)$. 因 $F^{2, m}$ 的正规正交 基为

$$
e_{n}=\sqrt{\frac{m !}{(n+m) !}} w^{n}, \quad n=0,1,2, \ldots
$$

故 $S e_{n}=(-r)^{n} e_{n}$, 且

$$
\sum_{n=0}^{\infty}\left\|S e_{n}\right\|_{2, m}^{2}=\sum_{n=0}^{\infty} r^{2 n}<\infty
$$

于是, $S$ 为 Hilbert-Schmidt 算子, 当然也是紧算子.

下面说明 $\left\|U_{z} S k_{z, m}\right\|_{p}$ 不是关于 $z \in \mathbb{C}$ 一致有界的. 由 $U_{z}, S$ 和 $k_{z, m}$ 的定义得

$$
\begin{aligned}
\mid U_{z} S k_{z, m} \|_{p}^{p} & =C(p, m) \int_{\mathbb{C}}\left|U_{z} S k_{z, m}\right|^{p} \mathrm{e}^{-|w|^{2}}|w|^{2 m} d A(w) \\
& =\frac{C(p, m)(m !)^{p}}{\mathrm{e}^{\left(\frac{1}{2}-r\right) p|z|^{2}}\left(\mathrm{e}^{|z|^{2}}-P_{m}\left(|z|^{2}\right)\right)^{\frac{p}{2}}} \int_{\mathbb{C}}\left|\mathrm{e}^{(1+r) w \bar{z}}-P_{m}\left(-r|z|^{2}+r w \bar{z}\right)\right|^{p} \mathrm{e}^{-|w|^{2}}|w|^{2 m} d A(w) .
\end{aligned}
$$


由不等式 $(a+b)^{p} \leqslant 2^{p}\left(a^{p}+b^{p}\right)$, 其中 $a, b>0, p>1$, 得

$$
\begin{aligned}
\mid U_{z} S k_{z, m} \|_{p}^{p} \geqslant & \frac{C(p, m)(m !)^{p}}{\mathrm{e}^{\left(\frac{1}{2}-r\right) p|z|^{2}\left(\mathrm{e}^{|z|^{2}}-P_{m}\left(|z|^{2}\right)\right)^{\frac{p}{2}}}} \\
& \times \int_{\mathbb{C}}\left(\frac{1}{2^{p}}\left|\mathrm{e}^{(1+r) p w \bar{z}}\right|-\left|P_{m}\left(-r|z|^{2}+r w \bar{z}\right)\right|^{p}\right) \mathrm{e}^{-|w|^{2}}|w|^{2 m} d A(w) \\
\geqslant & C_{1} \mathrm{e}^{p(1+r)|z|^{2}\left(\frac{p(1+r)}{4}-1\right)}-C_{2} Q_{m}\left(|z|^{2}\right),
\end{aligned}
$$

其中 $C_{1}$ 和 $C_{2}$ 为常数, 函数 $Q\left(|z|^{2}\right)$ 满足条件: 存在常数 $M$, 对任意 $z \in\{z \in \mathbb{C}:|z| \geqslant M\}$ 有 $Q\left(|z|^{2}\right) \leqslant|z|^{2 m p}$. 于是, 当 $|z| \rightarrow+\infty$ 时, $\mid U_{z} S k_{z, m} \|_{p}^{p} \rightarrow+\infty$. 这表明, 即使 $S$ 为 Hilbert-Schmidt 算子, $\left\|U_{z} S k_{z, m}\right\|_{p}$ 也不一定关于 $z$ 一致有界.

\section{3 紧性}

本节给出 $F^{2, m}$ 上算子为紧的两个充分条件. 下面先给出第一个条件.

定理 5 设 $S$ 为 $F^{2, m}$ 上线性算子且 $p>2$. 如果当 $z \rightarrow \infty$ 时, $\left\|U_{z} S k_{m, z}\right\|_{p, m} \rightarrow 0$, 那么, $S$ 为 $F^{2, m}$ 上紧算子.

证明 因当 $z \rightarrow \infty$ 时, $\left\|U_{z} S k_{m, z}\right\|_{p, m} \rightarrow 0$, 故 $\left\|U_{z} S k_{m, z}\right\|_{p, m}$ 关于 $z \in \mathbb{C}$ 一致有界. 于是, 由定理 4 得 $S$ 在 $F^{2, m}$ 上有界. 由引理 3 , 只需证明 $(2.1)$ 定义的算子 $T$ 为 $L^{2}\left(\mathbb{C}, d \lambda_{m}\right)$ 上紧算子. 下面使用逼 近方法证明 $T$ 的紧性.

对任意 $r>0$, 考查 $L^{2}\left(\mathbb{C}, d \lambda_{m}\right)$ 上算子 $T_{r}$, 其定义为

$$
T_{r} f(z)=\int_{|w|<r} f(w)\left\langle S K_{m, w}, K_{m, z}\right\rangle_{m} d \lambda_{m}(w)=\int_{\mathbb{C}} f(w) \chi_{r}(w)\left\langle S K_{m, w}, K_{m, z}\right\rangle_{m} d \lambda_{m}(w),
$$

其中 $\chi_{r}$ 是圆盘 $\{z \in \mathbb{C}:|z|<r\}$ 的特征函数. 由引理 2 易知,

$$
\int_{\mathbb{C}} \int_{\mathbb{C}}\left|\chi_{r}(w)\left\langle S K_{m, w}, K_{m, z}\right\rangle_{m}\right|^{2} d \lambda_{m}(z) d \lambda_{m}(w)<\infty .
$$

因此, $T_{r}$ 均为 Hilbert-Schmidt 算子. 于是 $T_{r}$ 均为紧算子.

设 $D_{r}=T-T_{r}$, 则

$$
D_{r} f(z)=\int_{\mathbb{C}} f(w)\left(1-\chi_{r}(w)\right)\left\langle S K_{m, w}, K_{m, z}\right\rangle_{m} d \lambda_{m}(w)=\int_{|w|>r} f(w)\left\langle S K_{m, w}, K_{m, z}\right\rangle_{m} d \lambda_{m}(w) .
$$

下面说明当 $r \rightarrow \infty$ 时, $\left\|D_{r}\right\| \rightarrow 0$, 这将说明 $T$ 为紧算子.

对任意 $\varepsilon>0$, 选择正数 $R$ 使得对全体 $|w|>R$ 有 $\left\|U_{w} S k_{m, w}\right\|_{p, m}<\varepsilon$. 由引理 2 , 对任意 $r>R$, $z, w \in \mathbb{C}$, 有

$$
\left|1-\chi_{r}(w)\right|\left|\left\langle S K_{m, w}, K_{m, z}\right\rangle_{m}\right| \leqslant \varepsilon|z-w|^{2 \sigma}|z|^{-m}|w|^{-m}\left(\mathrm{e}^{\frac{1}{2}\left(|z|^{2}+|w|^{2}\right)-\sigma|z-w|^{2}}+\mathrm{e}^{\frac{|z|^{2}}{2}-\sigma|z-w|^{2}} \sqrt{p_{m}\left(|w|^{2}\right)}\right)
$$

(上面可分别考虑 $|w| \leqslant r$ 和 $|w|>r$ ). 由定理 4 的证明知, 存在正常数 $C$, 它与 $\varepsilon$ 和 $r$ 均无关, 使得对 任意 $r>R$ 有 $\left\|D_{r}\right\| \leqslant C \varepsilon$. 这说明当 $r \rightarrow \infty$ 时, $\left\|D_{r}\right\| \rightarrow 0$.

因为每个 $U_{z}$ 为 $F^{2, m}$ 上西算子, 条件 $\left\|U_{z} S k_{m, z}\right\|_{2, m} \leqslant C$ 等价于条件 $\left\|S k_{z}\right\|_{2, m} \leqslant C$. 但是, $p \neq 2$ 时, $U_{z}$ 在 $L^{p}\left(\mathbb{C}, d \lambda_{m}\right)$ 上不是等距算子, 所以一个自然的想法就是去考查条件 $\left\|S k_{m, z}\right\|_{p, m} \leqslant C$, 这是第 二个关于紧性的条件. 
命题 6 设 $S$ 为 $F^{2, m}$ 上的线性算子且 $p>2$. 若存在常数 $C>0$, 对任意 $z \in \mathbb{C}$ 有 $\left\|S k_{m, z}\right\|_{p, m}$ $\leqslant C$ 且 $\left\|S^{*} k_{m, z}\right\|_{p, m} \leqslant C$, 则 $S$ 为 Hilbert-Schmidt 算子, 显然也是紧算子.

证明 由引理 1 和 $\left\|S k_{m, w}\right\|_{p, m}$ 的假设知, 存在常数 $C>0$ 使得

$$
\left|\left(S k_{m, z}\right)(z)\right| \leqslant C \mathrm{e}^{\frac{1}{p}|z|^{2}}|z|^{-\frac{2 m}{p}}, \quad z, w \in \mathbb{C} .
$$

上式可以改写为

$$
\left|\left\langle S K_{m, z}, K_{m, w}\right\rangle_{m}\right| \leqslant C \mathrm{e}^{\frac{1}{p}|z|^{2}}|z|^{-\frac{2 m}{p}}|w|^{-m}\left(\mathrm{e}^{\frac{1}{2}|w|^{2}}+\sqrt{p_{m}\left(|w|^{2}\right)}\right), \quad z, w \in \mathbb{C} .
$$

同样地, 关于 $\left\|S^{*} k_{w}\right\|_{p}$ 假设说明,

$$
\left|\left\langle S K_{m, w}, K_{m, z}\right\rangle_{m}\right| \leqslant C \mathrm{e}^{\frac{1}{p}|w|^{2}}|w|^{-\frac{2 m}{p}}|z|^{-m}\left(\mathrm{e}^{\frac{1}{2}|z|^{2}}+\sqrt{p_{m}\left(|z|^{2}\right)}\right), \quad z, w \in \mathbb{C} .
$$

将上面不等式 (3.1) 与 (3.2) 相乘再开平方得

$$
\left|\left\langle S K_{m, w}, K_{m, z}\right\rangle_{m}\right| \leqslant C \mathrm{e}^{\delta\left(|z|^{2}+|w|^{2}\right)}|z w|^{-2 m \delta} \sqrt{\left(1+\mathrm{e}^{-\frac{1}{2}|z|^{2}} p_{m}\left(|z|^{2}\right)\right)\left(1+\mathrm{e}^{-\frac{1}{2}|w|^{2}} p_{m}\left(|w|^{2}\right)\right)}, \quad z, w \in \mathbb{C},
$$

其中

$$
\delta=\frac{1}{2 p}+\frac{1}{4}<\frac{1}{2}
$$

于是,

$$
\int_{\mathbb{C}} \int_{\mathbb{C}}\left|\left\langle S K_{m, w}, K_{m, z}\right\rangle_{m}\right|^{2} d \lambda_{m}(w) d \lambda_{m}(z)<\infty,
$$

所以, 由下式定义的算子 $T$ :

$$
T f(z)=\int_{\mathbb{C}} f(w)\left\langle S K_{m, w}, K_{m, z}\right\rangle_{m} d \lambda_{m}(w)
$$

是 $L^{2}\left(\mathbb{C}, d \lambda_{m}\right)$ 上的 Hilbert-Schmidt 算子. 因为 $S$ 是 $T$ 在 $F^{2, m}$ 上的限制, 故 $S$ 是 Hilbert-Schmidt 算子.

在上面证明中只用了 $\left\|S k_{m, z}\right\|_{p, m}$ 和 $\left\|S^{*} k_{m, z}\right\|_{p, m}$ 逐点估计.

\section{4 具有无界符号的 $S_{p^{-}}$类 Toeplitz 算子}

为了构造 $F^{2, m}$ 上满足要求的 $S_{p^{-}}$类 Toeplitz 算子, 下面先引入一种以给定点 $\xi$ 为顶点的类圆雉 形集合. 对 $\delta>0, \xi \in \mathbb{C}$, 令

$$
\Omega(\xi, \delta)=\left\{z \in \mathbb{D}(0,|\xi|):\left[1-\left(1-\left|\frac{z}{\bar{\xi}}\right|\right)^{\delta}\right]^{\frac{1}{2}} \frac{|z-\xi|}{|\xi|}<\operatorname{Re}\left(\frac{\xi}{|\xi|} \frac{\overline{(\xi-z)}}{|\xi|}\right), \operatorname{Re}(z \bar{\xi})>0\right\},
$$

其中 $\mathbb{D}(0,|\xi|)$ 是以 0 点为心, $|\xi|$ 为半径的圆盘. 易知 $\Omega(\xi, \delta)$ 为 $\mathbb{D}(0,|\xi|)$ 中的开集, 它看起来像以 $\xi$ 为顶点的圆雉. 设 $\partial \mathbb{D}(0, r)$ 是圆盘 $\mathbb{D}(0, r)$ 的边界, $d s_{r}$ 为 $\partial \mathbb{D}(0, r)$ 上的 Lebesgue 弧长测度. 于是, $s_{r}(\partial \mathbb{D}(0, r))=O(r)$. 对任意 $\rho_{1}, \rho_{2}>0$, 可选择 $\delta=\delta\left(\rho_{1}, \rho_{2}\right)>0$ 使得对任意 $|\xi|$ 和 $0<r<|\xi|$ 有

$$
s_{r}\left(\Omega\left(\xi, \delta\left(\rho_{1}, \rho_{2}\right)\right) \cap \partial \mathbb{D}(0, r)\right)<\mathfrak{C}\left(|\xi|^{2}-r^{2}\right)^{\rho_{1}} \mathrm{e}^{-b_{2} r^{2}},
$$

其中 $\mathfrak{C}$ 是与 $\xi$ 和 $r$ 无关的常数. 为了方便, 后文将 $\Omega\left(\xi, \delta\left(\rho_{1}, \rho_{2}\right)\right)$ 写作 $\Omega\left(\xi, \rho_{1}, \rho_{2}\right)$. 
引理 7 设 $\rho_{1}, \rho_{2}>0$. 对任意 $\xi \in \mathbb{C}$, 设 $U_{\xi}(z)=\left(|\xi|^{2}-|z|^{2}\right)^{-\frac{\rho_{1}}{2}}$, 其中 $z \in \mathbb{D}(0,|\xi|)$, 且 $\chi_{\Omega\left(\xi, \rho_{1}, \rho_{2}\right)}(z)$ 为 $\Omega\left(\xi, \rho_{1}, \rho_{2}\right)$ 的特征函数. 令 $\psi=\chi_{\Omega\left(\xi, \rho_{1}, \rho_{2}\right)}(z) U_{\xi}(z)$, 则 $T_{\psi}$ 为 $F^{2, m}$ 上紧算子.

证明 因可将 Toeplitz 算子 $T_{\psi}$ 看作 $F^{2, m}$ 上以 $\bar{\psi} K_{m, z}$ 为核的积分算子, 故考虑积分

$$
J=\int_{\mathbb{C}}\left(\int_{\mathbb{C}}\left|\psi(w) K_{m, z}(w)\right|^{2} d \lambda_{m}(w)\right) d \lambda_{m}(z) .
$$

易知

$$
\begin{aligned}
J & =\int_{\mathbb{C}} \int_{\mathbb{C}}\left|\psi(w) K_{m, z}(w)\right|^{2} d \lambda_{m}(w) d \lambda_{m}(z)=\int_{\mathbb{C}}|\psi(w)|^{2}\left(\int_{\mathbb{C}}\left|K_{m, z}(w)\right|^{2} d \lambda_{m}(z)\right) d \lambda_{m}(w) \\
& =\int_{\mathbb{C}}|\psi(w)|^{2} \frac{\mathrm{e}^{|w|^{2}}-p_{m}\left(|w|^{2}\right)}{|w|^{2 m}} d \lambda_{m}(w)=\frac{1}{\pi m !} \int_{\mathbb{C}}|\psi(w)|^{2}\left(1-\mathrm{e}^{-|w|^{2}} p_{m}\left(|w|^{2}\right)\right) d A(w) \\
& =\frac{1}{\pi m !} \int_{\Omega\left(\xi, \rho_{1}, \rho_{2}\right)} U_{\xi}(w)\left(1-\mathrm{e}^{-|w|^{2}} p_{m}\left(|w|^{2}\right)\right) d A(w) \\
& =\frac{1}{\pi m !} \int_{\Omega\left(\xi, \rho_{1}, \rho_{2}\right)}\left(|\xi|^{2}-|w|^{2}\right)^{-\rho_{1}}\left(1-\mathrm{e}^{-|w|^{2}} p_{m}\left(|w|^{2}\right)\right) d A(w) \\
& =C_{1} \int_{0}^{|\xi|} \int_{\partial \mathbb{D}(0, r)}\left(|\xi|^{2}-|w|^{2}\right)^{-\rho_{1}}\left(1-\mathrm{e}^{-|w|^{2}} p_{m}\left(|w|^{2}\right)\right) d s_{r} d r \\
& \leqslant C_{2} \int_{0}^{|\xi|} s_{r}\left(\Omega\left(\xi, \rho_{1}, \rho_{2}\right)\right)\left(|\xi|^{2}-|w|^{2}\right)^{-\rho_{1}}\left|1-\mathrm{e}^{-|w|^{2}} p_{m}\left(|w|^{2}\right)\right| d r \\
& \leqslant C_{2} \mathfrak{C} \int_{0}^{|\xi|} \mathrm{e}^{-\rho_{2} r^{2}} d r=C_{2} \mathfrak{C} \frac{\sqrt{\rho_{2} \pi}}{2 \rho_{2}},
\end{aligned}
$$

其中 $C_{2} \mathfrak{C} \frac{\sqrt{\rho_{2} \pi}}{2 \rho_{2}}$ 为与 $\xi$ 无关的常数. 于是 $T_{\psi}$ 为紧算子.

众所周知, 容易找到复平面 $\mathbb{C}$ 的一个可数稠密子集 $\left\{\xi_{i}\right\}_{i=1}^{\infty}$. 由它的稠密性知, 可以构造函数 $\Psi$, 它在 $\mathbb{C}$ 的每一点为本性无界使得 Toeplitz 算子 $T_{\Psi}$ 为紧算子.

引理 8 存在函数 $\Psi \in L^{2}\left(\mathbb{C}, d \lambda_{m}\right)$, 它在 $\mathbb{C}$ 的每一点为本性无界, 使得 $T_{\Psi}$ 为 $F^{2, m}$ 上紧算子.

证明 对任意 $\xi \in \mathbb{C}$ 和 $r>0$, 下面说明 $\Psi$ 满足

$$
\operatorname{esssup}_{z \in \mathbb{D}(\xi, r)}|\Psi(z)|=\infty .
$$

这表明 $\Psi$ 在 $\mathbb{C}$ 的每一点为本性无界. 进而再证明 $T_{\Psi}$ 为紧算子.

设 $\rho_{1}, \rho_{2}>0$ 且 $U_{\xi_{i}}$ 为引理 7 中定义的函数. 由引理 7 , 对每个 $\psi_{i}(z)=\chi_{\Omega\left(\xi_{i}, \rho_{1}, \rho_{2}\right)}(z) U_{\xi_{i}}(z)$, Toeplitz 算子 $T_{\varphi_{i}}$ 为紧算子. 对每个 $f \in F^{2, m}$ 有

$$
\begin{aligned}
\left\|T_{\psi_{i}} f\right\|^{2} & =\int_{\mathbb{C}}\left|\int_{\mathbb{C}} \psi_{i}(w) f(w) \overline{K_{m, z}(w)} d \lambda_{m}(w)\right|^{2} d \lambda_{m}(z) \\
& =\int_{\mathbb{C}}\left|\int_{\Omega\left(\xi_{i}, \rho_{1}, \rho_{2}\right)}\left(\left|\xi_{i}\right|^{2}-|w|^{2}\right)^{-\frac{\rho_{1}}{2}} f(w) \overline{K_{m, z}(w)} d \lambda_{m}(w)\right|^{2} d \lambda_{m}(z) \\
& \leqslant\|f\|_{2, m}^{2} \int_{\mathbb{C}} \int_{\Omega\left(\xi_{i}, \rho_{1}, \rho_{2}\right)}\left(\left|\xi_{i}\right|^{2}-|w|^{2}\right)^{-\rho_{1}}\left|K_{z}(w)\right|^{2} d \lambda_{m}(w) d \lambda_{m}(z) \\
& \leqslant C_{1} \mathfrak{C} \frac{\sqrt{\rho_{2} \pi}}{2 \rho_{2}}\|f\|_{2, m}^{2}=\mathfrak{C}_{1}\|f\|_{2, m}^{2},
\end{aligned}
$$

其中 $\mathfrak{C}_{1}>0$ 为与 $\xi_{i}$ 无关的常数. 故 $\left\|T_{\psi_{i}}\right\| \leqslant \sqrt{\mathfrak{C}_{1}}$. 
对任意正整数 $I$, 设 $\psi_{I}=\sum_{i=1}^{I} \frac{1}{2^{i}} \psi_{i}$, 则对任何 $J>I$ 有

$$
\left\|T_{\psi_{J}}-T_{\psi_{I}}\right\|=\left\|\sum_{i=I+1}^{J} \frac{1}{2^{i}} T_{\psi_{i}}\right\| \leqslant \sum_{i=I+1}^{J} \frac{1}{2^{i}}\left\|T_{\psi_{i}}\right\| \leqslant \sqrt{\mathfrak{C}_{1}} \sum_{i=I+1}^{J} \frac{1}{2^{i}} .
$$

故 $\sum_{i=1}^{\infty} \frac{1}{2^{i}} T_{\psi_{i}}$. 按范数收玫到 $T$, 所以, $T$ 是紧算子.

由直接计算得

$$
\begin{aligned}
\left\|\psi_{i}\right\|_{2, m}^{2} & =\int_{\mathbb{C}}\left|\psi_{i}\right|^{2} d \lambda_{m}=\int_{\Omega\left(\xi_{i}, \rho_{1}, \rho_{2}\right)}\left(\left|\xi_{i}\right|^{2}-|w|^{2}\right)^{-\rho_{1}} d \lambda_{m}(w) \\
& =C_{1} \int_{0}^{\left|\xi_{i}\right|} \int_{\Omega\left(\xi_{i}, \rho_{1}, \rho_{2}\right) \cap \partial \mathbb{D}(0, r)}\left(\left|\xi_{i}\right|^{2}-r^{2}\right)^{-\rho_{1}} \mathrm{e}^{-r^{2}} r^{2 m} d s_{r} d r \\
& \leqslant C_{1} \mathfrak{C} \int_{0}^{\left|\xi_{i}\right|} \mathrm{e}^{-\left(1+\rho_{2}\right) r^{2}} r^{2 m} d r \\
& \leqslant C_{1} \mathfrak{C} \int_{0}^{\infty} \mathrm{e}^{-\rho_{2} r^{2}} d r=\mathfrak{C}_{1} .
\end{aligned}
$$

故

$$
\sum_{i=1}^{\infty} \frac{1}{2^{i}} \psi_{i} \rightarrow \Psi \in L^{2}\left(\mathbb{C}, d \lambda_{m}\right)
$$

对任意解析多项式 $p$, 当 $I \rightarrow \infty$ 时,

$$
\begin{aligned}
\left\|\left(T_{\Psi}-T_{\psi_{I}}\right) p\right\|_{2} & =\left\|\left(T_{\sum_{i=I+1}^{\infty} \frac{1}{2^{i}} \psi_{i}}\right) p\right\|_{2, m}=\left\|\left(\sum_{i=I+1}^{\infty} \frac{1}{2^{i}} T_{\psi_{i}}\right) p\right\|_{2, m} \leqslant \sum_{i=I+1}^{\infty} \frac{1}{2^{i}}\left\|T_{\psi_{i}} p\right\|_{2, m} \\
& \leqslant \sum_{i=I+1}^{\infty} \frac{1}{2^{i}} \sqrt{\mathfrak{C}_{1}}\|p\|_{2, m} \sum_{i=I+1}^{\infty} \frac{1}{2^{i}} \rightarrow 0 .
\end{aligned}
$$

这说明 $T=T_{\Psi}$. 因 $\left\{\xi_{i}\right\}_{i=1}^{\infty}$ 为 $\mathbb{C}$ 的稠密子集, 于是, 对任意 $\xi, r>0$,

$$
\operatorname{esssup}_{z \in \mathbb{D}(\xi, r)}|\Psi(z)|=\infty \text {. }
$$

引理证毕.

接下来将对 $0<p<\infty$ 构造 $F^{2, m}$ 上的 $S_{p^{-}}$类 Toeplitz 算子, 它的符号在 $\mathbb{C}$ 的每一点本性无界.

定理 9 存在函数 $\Psi \in L^{2}\left(\mathbb{C}, d \lambda_{m}\right)$, 它在 $\mathbb{C}$ 的每点本性无界, 使得 $T_{\Psi}$ 为 $F^{2, m}$ 上的 $S_{p^{-}}$类算子, 其中 $0<p<\infty$.

证明 设 $e_{k}(z)=\sqrt{\frac{m !}{(k+m) !}} z^{k}$, 则 $e_{k}$ 为 $F^{2, m}$ 的正规正交基. 对 $\rho_{1}, \rho_{2}>0$ 和任意正整数 $i$, 若 $\left|\xi_{i}\right|>1$, 对 $\gamma_{i} \in\left[\sqrt{\left|\xi_{i}\right|^{2}-1},\left|\xi_{i}\right|\right)$, 令 $\psi_{i}(z)=\chi_{\Omega_{\gamma_{i}}\left(\xi_{i}, \rho_{1}, \rho_{2}\right)} U_{\xi_{i}}$, 其中 $\Omega_{\gamma_{i}}\left(\xi_{i}, \rho_{1}, \rho_{2}\right)=\left\{z \in \Omega\left(\xi_{i}, \rho_{1}, \rho_{2}\right)\right.$ : $\left.|z|>\gamma_{i}\right\}$; 若 $\left|\xi_{i}\right| \leqslant 1$, 令 $\psi_{i}(z)=\chi_{\Omega\left(\xi_{i}, \rho_{1}, \rho_{2}\right)} U_{\xi_{i}}$, 其中 $U_{\xi_{i}}$ 是引理 8 中定义的函数.

于是, 对 $\left|\xi_{i}\right|>1$ 有

$$
\begin{aligned}
\left\langle T_{\psi_{i}} e_{k}, e_{k}\right\rangle_{m} & =\left\langle\psi_{i} e_{k}, e_{k}\right\rangle_{m}=\int_{\mathbb{C}} \psi_{i}(w)\left|e_{k}(w)\right|^{2} d \lambda_{m}(w) \\
& =\frac{m !}{(m+k) !} \int_{\mathbb{C}} \psi_{i}(w)|w|^{2 k} d \lambda_{m}(w)=\frac{m !}{(m+k) !} \int_{\Omega\left(\xi_{i}, \rho_{1}, \rho_{2}\right)}\left(\left|\xi_{i}\right|^{2}-|w|^{2}\right)^{-\frac{\rho_{1}}{2}}|w|^{2 k} d \lambda_{m}(w) \\
& \leqslant C_{1} \frac{m !}{(m+k) !} \int_{\gamma_{i}}^{\left|\xi_{i}\right|} \int_{\Omega\left(\xi_{i}, \rho_{1}, \rho_{2}\right) \cap \partial \mathbb{D}(0, r)}\left(\left|\xi_{i}\right|^{2}-r^{2}\right)^{-\frac{\rho_{1}}{2}} r^{2 k+2 m} \mathrm{e}^{-r^{2}} d A(w)
\end{aligned}
$$




$$
\begin{aligned}
& \leqslant C_{1} \frac{m !}{(m+k) !} \int_{\gamma_{i}}^{\left|\xi_{i}\right|}\left(\left|\xi_{i}\right|^{2}-r^{2}\right)^{\frac{\rho_{1}}{2}} \mathrm{e}^{-\left(\rho_{2}+1\right) r^{2}} r^{2 k+2 m} d r \\
& \leqslant C_{1} \frac{m !}{(m+k) !} \int_{\gamma_{i}}^{\left|\xi_{i}\right|} \mathrm{e}^{-\left(\rho_{2}+1\right) r^{2}} r^{2 k+2 m} d r \leqslant C_{1} \frac{m !}{(m+k) !} \int_{0}^{\infty} \mathrm{e}^{-\left(\rho_{2}+1\right) r^{2}} r^{2 k+2 m} d r \\
& =C_{1} \frac{m !}{(m+k) !} \int_{0}^{\infty} \mathrm{e}^{-\left(\rho_{2}+1\right) r^{2}} r^{2 k+2 m-1} d r^{2} \\
& =C_{1} \frac{m !}{2(m+k) !} \frac{1}{\left(\rho_{2}+1\right)^{k+m+\frac{1}{2}}} \int_{0}^{\infty} \mathrm{e}^{-\left(\rho_{2}+1\right) r^{2}}\left(\left(\rho_{2}+1\right) r^{2}\right)^{k+m-\frac{1}{2}} d\left[\left(\rho_{2}+1\right) r^{2}\right] \\
& =C_{1} \frac{m !}{2(m+k) !} \frac{1}{\left(\rho_{2}+1\right)^{k+m+\frac{1}{2}}} \Gamma\left(k+m+\frac{1}{2}\right) .
\end{aligned}
$$

同理, 若 $\left|\xi_{i}\right| \leqslant 1$, 则

$$
\left\langle T_{\psi_{i}} e_{k}, e_{k}\right\rangle_{m} \leqslant C_{1} \frac{m !}{2(m+k) !} \frac{1}{\left(\rho_{2}+1\right)^{k+m+\frac{1}{2}}} \Gamma\left(k+m+\frac{1}{2}\right) .
$$

由 Stirling 公式得

$$
C_{1} \frac{m !}{2(m+k) !} \frac{1}{\left(\rho_{2}+1\right)^{k+m+\frac{1}{2}}} \Gamma\left(k+m+\frac{1}{2}\right) \sim \mathfrak{C}_{2} \frac{1}{\left(\rho_{2}+1\right)^{k+m+\frac{1}{2}}} \frac{1}{\sqrt{k+1}},
$$

其中 $\mathfrak{C}_{2}$ 是与 $\xi_{i}$ 和 $k$ 无关的常数.

实际上, 只需考虑 $0<p \leqslant 1$ 的情形. 因 $T_{\Psi}=T_{\sum_{i=1}^{\infty} \frac{1}{2^{i}} \psi_{i}}$ 为正算子, 故

$$
\begin{aligned}
\sum_{k=0}^{\infty}\left\langle T_{\Psi} e_{k}, e_{k}\right\rangle_{m}^{p} & =\sum_{k=0}^{\infty}\left(\sum_{i=1}^{\infty} \frac{1}{2^{i}}\left\langle T_{\psi_{i}} e_{k}, e_{k}\right\rangle_{m}\right)^{p} \leqslant \sum_{k=0}^{\infty} \sum_{i=1}^{\infty} \frac{1}{2^{i p}}\left\langle T_{\psi_{i}} e_{k}, e_{k}\right\rangle_{m}^{p} \\
& \leqslant \mathfrak{C}_{3} \sum_{k=0}^{\infty} \sum_{i=1}^{\infty} \frac{1}{2^{i p}} \frac{1}{\left(\rho_{2}+1\right)^{p\left(k+m+\frac{1}{2}\right)}}\left(\frac{1}{k+1}\right)^{\frac{p}{2}} \\
& =\mathfrak{C}_{4} \sum_{k=0}^{\infty}\left(\frac{1}{\left(\rho_{2}+1\right)^{p}}\right)^{k+m+\frac{1}{2}}\left(\frac{1}{k+1}\right)^{\frac{p}{2}}<\infty
\end{aligned}
$$

其中 $\mathfrak{C}_{3}$ 和 $\mathfrak{C}_{4}$ 为常数. 于是 $T_{\Psi}$ 是 $S_{p^{-}}$类 Toeplitz 算子. 若 $1<p<\infty$, 显然 $T_{\Psi} \in S_{p}$.

\section{参考文献}

1 Cho H R, Zhu K. Fock-Sobolev spaces and their Carleson measures. ArXiv:1212.0737, 2012

2 Berger C, Coburn L. Toeplitz operators and quantum mechanics. J Funct Anal, 1986, 68: 273-299

3 Berger C, Coburn L. Toeplitz operators on the Segal-Bargmann space. Trans Amer Math Soc, 1987, 301: 813-829

4 Berger C, Coburn L. Heat flow and Berezin-Toeplitz estimates. Amer J Math, 1994, 116: 563-590

5 Coburn L, Isralowitz J, Li B. Toeplitz operators with BMO symbols on the Segal-Bargmann space. Tran Amer Math Soc, 2011, 363: 3015-3030

6 Isralowitz J, Zhu K. Toeplitz operators on the Fock space. Integral Equations Operator Theory, 2012, 72: 363-392

7 Zhu K H. Analysis on Fock space. New York: Springer-Verlag, 2012

8 Schuster A P, Varolin D. Toeplitz operators and Carleson measures on generalized Bargman-Fock spaces. Integral Equations Operator Theory, 2010, 66: 593-611

9 Axler S, Zheng D. Compact operators via Berezin tranforms. Indiana Univ Math J, 1998, 47: 387-400

10 Cao G, Wang X, Zhu K. Boundedness and compactness of operators on the Fock space. Integral Equations Operator Theory, 2013, 77: 355-370 
11 Dieudonne A, Tchoundja E. Toeplitz operators with $L^{1}$ symbols on Bergman spaces in the unit ball of $\mathbb{C}^{n}$. Adv Pure Appl Math, 2010, 2: 65-88

12 Miao J, Zheng D. Compact operators on Bergman spaces. Integral Equations Operator Theory, 2004, 48: 61-79

13 Zorboska N. Toeplitz operators with BMO symbols and the Berezin transform. Int J Math Sci, 2003, 46: 2926-2945

14 Cima J, Cuckovic Z. Compact Toeplitz operators with unbounded symbols. J Operator Theory, 2005, 53: 431-440

15 Cao G F. Toeplitz operators with unbounded symbols of several complex variables. J Math Anal Appl, 2008, 339: $1277-1285$

16 Xia J, Wang X, Cao G. $S_{p}$-class $(0<p<\infty)$ Toeplitz operators with unbounded symbols on Dirichlet space. Acta Sci Math, 2012, 32: 1919-1928

17 Cho H R, Zhu K. Fock-Sobolev spaces and their Carleson measures. J Funct Anal, 2012, 263: 2483-2506

18 Janson S, Peetre J, Rochberg R. Hankel forms and the Fock space. Rev Mat Iberoamericana, 1987, 3: 61-138

19 Zhu K H. Operator Theory in the Function Spaces, 2nd ed. Mathematical Surveys and Monographs, vol. 138. Providence, RI: Amer Math Soc, 2007

\section{Bounded, compact and $S_{p}$-class operators on Fock-Sobolev spaces}

\section{WANG XiaoFeng, XIA Jin \& CAO GuangFu}

Abstract The bounded and compactness for the densely-defined operators on the Fock-Sobolev space are discussed. We represent these operators an integral operators, then obtain sufficient conditions for boundedness of these operators. An example is given to show that these conditions are not necessary. Two conditions for compactness of these operators are given. the sufficient conditions of those two properties are given in this paper. The compact and even $S_{p}$-class $(0<p<\infty)$ Toeplitz operators on Fock-Sobolev space with symbols which are essential unbounded everywhere on complex plane are also constructed.

Keywords Fock-Sobolev space, Toeplitz operator, boundedness, compactness, Schatten $p$-class

MSC(2010) 30H20, 47B07, 47B35, 47B38

doi: $10.1360 / 012014-15$ 